congruence $1^{p-2}+2^{p-2}+\cdots+\left(\frac{p-1}{2}\right)^{p-2} \equiv 0(\bmod p)$. Professor Putnam discusses the general congruence of this type, $1^{r}+2^{r}+\cdots+\left(\frac{p-1}{2}\right)^{r} \equiv a(\bmod p)$, and shows that by expressing $a$ as a fraction it may be given a value for any fixed $r$ (less than $p$ ) that is independent of $p$.

5. In a paper in the Proceedings of the Edinburgh Mathematical Society, Professor Allardice considered a geometrical transformation in the plane, $\tan \frac{1}{2} \varphi=k \tan \frac{1}{2} \vartheta$, where $\vartheta$ is the angle formed by the enveloping tangents of a curve with a given straight line $l$, the axis of transformation, and $\varphi$ is the angle formed by $l$ and $t$, a system of lines through the intersection of $l$ and $t$, which envelop the transformed curve of c. In the present paper, Dr. Stager considers analytically a similar transformation in space and applies it to certain systems of spheres. The method of transformation applied to space is as follows: Let $\alpha$ be a given plane and $P$ be any solid. Further, let a plane $\beta$ be tangent to $P$ and intersect $\alpha$ in $i$, making with $\alpha$ an angle $\vartheta$. If through $i$ we draw a plane $\beta^{\prime}$, making with $\alpha$ an angle $\varphi$, such that $\tan \frac{1}{2} \varphi=k \tan \frac{1}{2} \vartheta$, the envelop of $\beta^{\prime}$ is defined as the "transform of $P$." The paper concludes with a number of applications of the method.

W. A. Manning,

Secretary of the Section.

THE TOTAL VARIATION IN THE ISOPERIMETRIC PROBLEM WITH VARIABLE END POINTS.

BY DR. A. R. CRATHORNE.

(Read before the Chicago Section of the American Mathematical Society, March 22, 1913.)

IN the simple problem of the calculus of variations,

$$
J=\int_{x_{1}}^{x_{2}} F\left(x, y, x^{\prime}, y^{\prime}\right) d t=\text { minimum, }
$$

the total variation can be expressed as an integral of which the integrand is the Weierstrassian $E$-function. It is the object of this note to express in a similar way the total vari- 
ation for those isoperimetric problems in which the so called Weierstrassian construction is possible.

Let $k_{1}: x=x_{1}(u), y=y_{1}(u)$ and $k_{2}: x=x_{2}(v), y=y_{2}(v)$ be two given curves lying wholly or partly in the region $R$ of the problem but not intersecting in this region. The calculus of variations problem under discussion is

while

$$
J=\int_{k_{1}}^{k_{2}} F\left(x, y, x^{\prime}, y^{\prime}\right) d t=\min .
$$

$$
K=\int_{k_{1}}^{k_{2}} G\left(x, y, x^{\prime}, y^{\prime}\right) d t=l .
$$

Suppose that $C_{0}: x=x(t), y=y(t)$ is a minimizing curve intersecting the curves $k_{1}$ and $k_{2}$ in 1 and 2 (figure). $C_{0}$

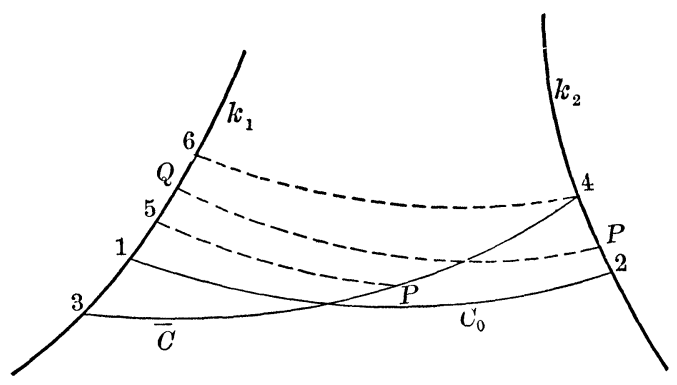

will be one of a two parameter family of extremals which are solutions of the Euler's equation for the problem

$$
J=\int H\left(x, y, x^{\prime}, y^{\prime}\right) d t=\min ., \quad H=F+\lambda G,
$$

and which intersect $k_{1}$ transversally. Let the equations of this family be

$$
x=\varphi(u, \lambda, t), \quad y=\psi(u, \lambda, t),
$$

intersecting $k_{1}$ for $t=t_{1}$. From these we have

$$
C_{0}: \quad x=x(t)=\varphi\left(u_{0}, \lambda_{0}, t\right), \quad y=y(t)=\psi\left(u_{0}, \lambda_{0}, t\right) .
$$

Let

$$
\chi(u, \lambda, t)=\int_{k_{1}}^{t} G\left(x, y, x^{\prime}, y^{\prime}\right) d t
$$


and consider in the usual way the space curves

Since

$$
x=\varphi(u, \lambda, t), \quad y=\psi(u, \lambda, t), \quad z=\chi(u, \lambda, t) .
$$

$$
\chi\left(u, \lambda, t_{1}\right)=0
$$

these curves will all intersect $k_{1}$ and they can be shown to fill a portion of space and, with the exception of points on $k_{1}$, to form a field $R^{\prime}$. Let $C_{0}^{\prime}$ be the space corresponding to $C_{0}$. Suppose $\bar{C}: x=\bar{x}(\tau), y=\bar{y}(\tau)$ to be any other curve connecting $k_{1}$ with $k_{2}$ for which $K=l$. Let $\bar{C}^{\prime}$ be the corresponding space curve which, since the Weierstrassian construction is possible, will lie in $R^{\prime}$. The equations of $\bar{C}^{\prime}$ are

$$
\begin{gathered}
x=\bar{x}(\tau), \quad y=\bar{y}(\tau), \\
z=\bar{z}(\tau)=\int_{\tau_{1}}^{\tau} G\left(\bar{x}, \bar{y}, \bar{x}^{\prime}, \bar{y}^{\prime}\right) d t .
\end{gathered}
$$

Now let the point $P$ (figure) move from 3 to 2 , taking the path 34 along $\bar{C}$, then 42 along $k_{2}$. First considering $P$ as a point on $\bar{C}$, there will be one extremal $5 P$ through $P$ cutting $k_{1}$ transversely for which $K_{5 P}=\bar{K}_{3 P}$. We consider in the usual way* $J_{5 P}+\bar{J}_{P 4}$, and find

$$
J_{\bar{C}}-J_{64}=\int_{\tau_{3}}^{\tau_{4}} E d \tau .
$$

Following the point $P$ from 4 to 2 along $k_{2}$, consider the integral $J$ along the extremals which cut $k_{1}$ transversely and for which $K=l$. Let $Q$ be the intersection of these extremals with $k_{1}$, then as $P$ moves from 4 to $2, Q$ will move from 6 to 1 . The integral $J_{Q P}$ is a function of the parameter $v$, and using the field integral notation, $\dagger$

$$
J_{Q P}=W\left(x_{2}(v), y_{2}(v), z\right) .
$$

Since $z$ is constant and equal to $l$,

$$
\frac{d J_{Q P}}{d v}=x_{2}{ }^{\prime} W_{x_{2}}+{y_{2}}^{\prime} W_{y_{2}}=x_{2}{ }^{\prime} H_{x^{\prime}}+y_{2}{ }^{\prime} H_{y^{\prime}}
$$

The arguments of $H_{x^{\prime}}$ and $H_{y^{\prime}}$ are $x_{2}(v), y_{2}(v)$, and $x^{\prime}$ and $y^{\prime}$

* Bolza, Variationsrechnung, p. 507.

† Ibid., p. 504 . 
for the extremal $Q P$ at its intersection with $k_{2}$. Let $J_{Q P}=S(v)$; then

$$
\begin{gathered}
J_{64}=S\left(v_{4}\right), \quad J_{c_{0}}=J_{12}=S\left(v_{2}\right), \\
J_{64}-J_{c_{0}}=-\left[S\left(v_{2}\right)-S\left(v_{4}\right)\right]=-\int_{v_{4}}^{v_{2}} \frac{d S}{d v} d v \\
=-\int_{v_{4}}^{v_{2}}\left[x_{2}{ }^{\prime} H_{x^{\prime}}+y_{2}{ }^{\prime} H_{y^{\prime}}\right] d v,
\end{gathered}
$$

and we have for the total variation

$$
\Delta J=J_{\bar{c}}-J_{c_{0}}=\int_{\tau_{3}}^{\tau_{4}} E d \tau-\int_{v_{4}}^{v_{2}}\left[x_{2}{ }^{\prime} H_{x^{\prime}}+y_{2}{ }^{\prime} H_{y^{\prime}}\right] d v .
$$

At the point $2, C_{0}$ cuts $k_{2}$ transversally, that is,

$$
x_{2}{ }^{\prime}(v) H_{x^{\prime}}+\left.y_{2}{ }^{\prime}(v) H_{y^{\prime}}\right|^{2}=0,
$$

and in this problem, as in the simple problem of the calculus of variations, we are led to a study of the sign of $E$ along the extremal $C_{0}$ when considering sufficient conditions.

UNIVERSITY OF IllinoIs, March, 1913.

\section{A NOTE ON GRAPHICAL INTEGRATION OF A FUNCTION OF A COMPLEX VARIABLE.}

BY DR. S. D. KILLAM.

(Read before the American Mathematical Society, April 26, 1913.)

THE object of this paper is to give a shorter and purely graphical method for graphical integration than that of the author in his thesis* on graphical integration of functions of a complex variable.

We can represent a function $f(z)$ of the complex variable $z=r e^{i \theta}$ in the $f(z)$-plane by a system of orthogonal curves $r=r_{n}(n=0,1, \cdots, n)$ and $\theta=\theta_{n}(n=0,1, \cdots, n)$. We choose the values $r_{n}$ and $\theta_{n}$ so that the $f(z)$ plane is covered by a net of small squares. We seek now a graphical representation in the $Z=X+i Y$-plane of the function $Z=\int_{0}^{z_{n}} f(z) d z$,

* " Über graphische Integration von Funktionen einer complexen Variabeln mit speziellen Anwendungen," Dissertation, Göttingen, 1912. Referred to in this paper as "thesis." 